\title{
Sequential fragmentation: The origin of columnar quasi-hexagonal patterns
}

\author{
E. A. Jagla ${ }^{1}$ and A. G. Rojo ${ }^{2}$ \\ ${ }^{1}$ Centro Atómico Bariloche and Instituto Balseiro, Comisión Nacional de Energía Atómica \\ (8400) Bariloche, Argentina \\ 2 Department of Physics, The University of Michigan \\ Ann Arbor, Michigan, 48109-1120, USA
}

\begin{abstract}
We present a model that explains the origin and predicts the statistical properties of columnar quasi-hexagonal crack patterns, as observed in the columnar jointing of basaltic lava flows. Irregular fractures appear at the surface of the material, induced by temperature gradients during cooling. At later times fractures penetrate into the material, and tend to form polygonal patterns. We show that this ordering can be described as a tendency to minimize an energy functional. Atomistic simulations confirm this interpretation. Numerical simulations based on a phenomenological implementation of this principle generate patterns that have remarkably good statistical agreement with real ones.
\end{abstract}

\section{INTRODUCTION}

Columnar jointing in some kinds of volcanic rocksespecially basaltic lava flows-is one spectacular example of geometrical order in nature, where cracks split the rock in a set of parallel columns [1,221. Perpendicularly to the columns the fractures show a distinctive pattern of mostly pentagonal and hexagonal polygons whose sizes vary from a few centimeters to about two meters (see Fig. 11). It has been realized for more than a century that columns result from the contraction of the cooling lava after solidification. There is consensus by now that fractures start at the surfaces of the igneous body, and propagate to the interior as the rock cools down. Fracture patterns at the surface are rather disordered, but the fractures progressively order as they penetrate the sample, reaching an almost stable polygonal configuration after some depth. The fundamental reason of this ordering process is unknown at present. Further evidence for these facts come from the reproduction of columnar cracking in samples of dessicating starches [3]. In this case the columns have diameters in the range of the millimeters, and the ordering process is apparent.

Ryan and Sammis [1] collected evidence suggesting that the vertical advance of the fracturing front is not continuous, but a discrete process that we call sequential fragmentation: at each step, and when some maximum tensile stress is exceeded, a layer of material is fractured. The existence of this punctuated advance of the fracturing front can be seen in the lateral faces of the columns, which usually have typical marks called striae, or chisel marks 4. Further work [5,6] has clarified the form in which fractures propagate within each horizontal layer. A fracture appears at some point and propagates under the combination of mode I and mode III fracturing, guided by the upper part of the rock, which was fractured previously. This is a justification for the prismatic form of the columns and the striae on their faces. However, it assumes that the polygonal pattern is already formed in the upper parts of the rock. Aydin and DeGraff [6] tried to give a justification for the evolution of superficial fractures, which usually meet in the form of a ' $\mathrm{T}$ ', towards the ' $\mathrm{Y}$ ' triple junctions typical of well developed columns. But, as they point out, the prediction of the overall polygonal pattern of fractures would require a three dimensional mechanical analysis of the interaction among many neighbor triple junctions. This is a extremely difficult task if pursuit from a microscopic point of view. They conclude that probably energetic arguments (involving fracture energy and elastic energy) may dictate the way in which the final polygonal pattern is formed.

Energetic arguments have been invoked since quite a long time to justify the polygonal structure of columnar basalts [7], and it is known that the perfect hexagonal pattern relieves the maximum amount of elastic energy for a given total length of fractures [8]. We argue in the next section that for the problem of sequential fragmentation (in which new parts of the rock are sequentially fractured under the influence of both the previously fractured parts and the still intact rock underneath) a minimum principle can be invoked to describe the evolution in depth of the fracture pattern. In the next section we actually show in a numerical simulation (which assumes only the sequential nature of the fragmentation process and no other ad-hoc assumption) that the fracture pattern starts being disordered at the surface, and progressively orders as it penetrates the sample, approaching the ideal pattern we expect on an energetic basis. The minimum principle is used in section III in phenomenological simulations, to evolve superficially disordered patterns into stable polygonal configurations. The statistical properties of the final patterns agree with the available experimental data in basalts and also in starches. In section IV we summarize our results, and point out some open problems in columnar jointing, mainly associated to realistic conditions of cooling. 


\section{ENERGETIC DESCRIPTION OF THE ORDERING PROCESS}

We will concentrate on the problem of a semi-infinite solid body ('the rock') cooling down through a (horizontal) free surface [9]. Since this is a situation of inhomogeneous cooling, there will be thermal gradients within the rock. Thermal gradient will point vertically at every point, and then temperature will be constant in any horizontal plane. Under the stresses generated by the thermal gradient, the rock will fracture.

There are two qualitatively different stages in the fracturing process. One is the appearance of fractures at the surface of the rock. Here, the first fracture appears when some maximum stress is exceeded at some point of the sample. Then it propagates horizontally under the influence of the inhomogeneities of the rock. When new fractures nucleate at the surface, they propagate until they meet older ones, usually at right angles, giving rise to typical surface fragmentation patterns that have been extensively studied, both experimentally [10,11] and theoretically 12,13]. For our purposes, we only mention that this stage is governed to a large extent by the random disorder present in the system, since fractures nucleate at points where the body can resist the lowest strain. The pattern at the surface is usually quite disordered.

In this paper we study the second stage of the fracturing process of the rock, namely the way in which the superficial, disordered pattern of fractures penetrates the body and orders. We will assume that the temperature distribution within the rock is a given function of coordinates and time, independent of the actual arrangement of fractures, and homogeneous at each horizontal plane. The last fact, however, is not enough to assure that the fracture front (i.e, the vertical coordinate up to which fractures have penetrated, as a function of the horizontal coordinate) will be horizontal. In fact, in a standard situation of fracture mechanics [14], it would occur that as soon as a fracture penetrates slightly more than the rest, stresses accumulate onto that fracture, the result being that typically a single fracture advances. For our case however, and under realistic cooling conditions, the temperature gradient decreases ahead of the fractures, so if a fracture advances, it rapidly reaches regions where the lower temperature gradient precludes the further advance of that fracture. This is the reason for the sequential advance of the fracture front as schematically illustrated in Fig. 2. At each "time step", a horizontal slab of material right below the fracture front is fractured under the influence of the already fractured material above, and the still unfractured material below. We will always assume that the conditions for sequential fragmentation apply.

¿From now on we will treat the rock as a collection of particles, elastically joined to their nearest neighbors, in the presence of a constant temperature gradient in the vertical direction. Changes in temperature are interpreted as changes in the equilibrium distance between particles. Fractures will be modeled by the saturation of the elastic energy between neighbor particles as they are taken apart a distance larger than some pre-fixed value $d_{c r}(T)$ (see Fig. 3). Note that, in this way, for any given temperature distribution, and for any arrangement of particles, we can define a total energy $E$ for the system without ambiguity.

It is useful to divide the total energy $E$ in two parts, $E=E_{1}+E_{2}$. The $E_{1}$ term, that we call elastic energy, comes from those particles being at relative distance lower than $d_{c r}$. This is an elastic energy since is quadratic in the relative distance between particles. The second part $E_{2}$ is the contribution from particles at relative distance larger than $d_{c r}$, and then it can be associated with broken links (since in this case force vanishes), and identified with the fracture energy. Actually, whenever we talk of the existence of a fracture at a given position of the sample, we mean that neighbor particles are separated by a distance larger than $d_{c r}$ across that 'fracture'.

Having defined the degrees of freedom of the system and the total energy, we can think of the system as a point $\mathcal{P}$ in the configuration space of all particle coordinates. The sequential evolution we have described (Fig. 2), corresponds to the sequential mechanical relaxation of all particles within the slab between $z_{i}$ and $z_{i+1}$, with $d z=z_{i+1}-z_{i}$ being the thickness of the slab being fractured at step $i$. This sequential process corresponds to the movement of $\mathcal{P}$ in the energy landscape. Assuming that the mechanical relaxation occurs by some kind of 'viscous' dynamics, the present description becomes complete and deterministic, and then we can solve in principle all the (non-linear) mechanical equations for the problem, and obtain in all detail the way in which fractures penetrate the sample. The qualitative features of this advance, however, can be inferred from general arguments. In fact, with the fracture front at a given $z$ position, we can calculate the stress field ahead of the fractures, and determine the directions along which this stress is maximized. These are the directions that fractures have the tendency to follow as they advance. The system releases the maximum amount of energy when fractures advance along these directions, compared to any other. In other words, at each step the configuration point $\mathcal{P}$ moves following a steepest descendent path in the potential energy landscape. Note that due to the particular conditions of sequential advance, this movement is 'quasistatic', in the sense that it does not involve the runaway of fractures ahead the fracture front.

The kind of argument we are using is equivalent to those used in surface fragmentation to justify the fact that new fractures meet older ones at right angles [2]. This is a consequence of the tendency of fractures to advance perpendicularly to the direction of maximum stress, and is equivalent to say that the configuration point $\mathcal{P}$ moves down in energy following the steepest descendent path. We are just saying that for sequential fragmentation the advance of all fractures is governed 
by this kind of principle. Then, our minimum principle, central to all this work, states that under sequential fragmentation conditions the advance of the fracture front occurs with a tendency to reduce as much as possible the total energy of the system. Note that during this ordering process, the existence of small inhomogeneities in the material plays no significant role, as energy will be mostly dependent only on the geometrical configuration of fractures.

Our principle then justifies qualitatively the observed tendency to produce polygonal arrangements. It is important to note, that the system finds the most convenient pattern by modifying the one at the surface (which usually is quite disordered) through small steps as fractures penetrate the sample. In the next two sub-sections we present results that confirm the validity of our interpretation.

\section{A. A stress calculation}

First of all we want to show that standard stresses calculations are consistent with the minimum principle. We have calculated the stress field surrounding a system of unevenly spaced fractures in two dimensions. More specifically, we want to calculate the stress field for a set of fractures as depicted in the inset of Fig. 1, namely, there are pairs of fractures separated by a distance $d_{1}$, and the pairs themselves are separated by some other distance $d_{2}$.

We start with lattice points joined by springs to form a triangular lattice, then modeling an homogeneous and isotropic material with Poisson ratio 15$] \sigma=2 /(4+$ $\sqrt{3}) \sim 0.35$. We simulate a piece of size $l_{x} \times l_{z}$ in the $x$ and $z$ directions respectively, taking periodic boundary conditions in the $x$ direction, and open boundary conditions in the $z$ direction. The springs have a rest length $d_{0}$ that depends on its vertical coordinate $z$ according to

$$
d_{0}(z)=d_{00}\left(1-\beta \frac{z}{l_{z}}\right)
$$

In this way we model a constant temperature gradient in the $z$ direction (in the simulations we will use $\beta=0.01$ ). The periodic boundary conditions in the $x$ direction are taken in such a way that the particles at $z=0$ are nominally at zero strain, whereas all planes on top of that are strained with respect to the preferred distance $d_{0}$. The two fractures are introduced in the system by eliminating all springs that go across the fractures.

We have solved numerically the problem, by relaxing (with a viscous dynamics) the coordinates of the particles in order to obtain the equilibrium configuration. Then the stress tensor [15] was calculated and diagonalized at each position. In Fig. 4 we show the results. At each point, the tangent to the line shown in that figure is the direction perpendicular to the eigenvector corresponding to the maximum eigenvalue of the stress tensor, and then it is the direction that fractures will tend to follow as they advance. Starting at the tips of the fractures, we see that these directions go away from each other, as indicated by the arrows. This indicates that, if sequential fragmentation occurs, the close fractures will advance with a tendency to separate from each other, and eventually to produce a set of evenly spaced fractures. In fact, only when the evenly spaced configuration is reached, the maximum stress direction will coincide with the vertical direction, and from here the pattern is not modified. This standard calculation coincides qualitatively with what expected from the minimum principle, since a set of evenly spaced fractures is the configuration that releases the maximum amount of energy (this is the equivalent of the honeycomb lattice in three dimensions [16]). Then we see that the conclusions from our minimum principle do not contradict those obtained from more standard analysis. The advantage, however, is that the minimum principle is much easier to implement in cases where a calculation of stresses is not feasible.

\section{B. Atomistic simulations of ordering}

The second result presented to validate the minimum principle is an atomistic numerical simulation in three dimensional systems. We implement sequential fragmentation in the following way. We use a generalization of the procedure extensively used to study surface fragmentation [12]. In that case a layer of material shrinks while it is attached to a fixed underlying layer. We take an hexagonal plane of particles, with particles attached to their neighbors by generalized springs (with an energydisplacement relation as that of Fig. (3) of spring constant $K$ and initial natural length $d_{0}$. Their positions are the dynamical variables. They are attached to an underlying hexagonal plane of particles (which are kept fixed to their original positions during the simulations) by vertical springs of constant $k$. The vertical springs do not break. Simulation proceeds by reducing the equilibrium distance of the horizontal springs of the layer being simulated. The first fracture appears when the equilibrium distance between two particles becomes grater than the corresponding critical distance $d_{c r}$ of the spring that joins them.

For our simulations of sequential fragmentation, the only difference is that we consider also the simulated plane to be joined to an upper plane of fixed particles by spring of constant $k$, and that we simulate the fracturing of the system as a sequence of independent two dimensional fragmentation processes. In the simulation of the successive layers the position of particles in the upper plane are taken equal to the final positions of the simulation of the previous layer. In the simulation of the first layer, we do not have an upper plane. However, to avoid introducing disorder into the system (and in order to break the homogeneity that would occur for an absolutely perfect system) we take an upper plane con- 
sisting of particles located at the hexagonal lattice plus some random displacement, independent for each particle. We took this displacement to be 0.5 of the lattice parameter. We want to mention that other simulations in which disorder was included, and the first layer was simulated without any upper plane produced qualitatively the same results. The equilibrium distance between particles $d_{0}$ within the layer being simulated is quasistatically reduced from some initial value $d_{00}$ to $p d_{00}$. We use $p=0.89$. In the energy of the horizontal springs (Fig. 3) we use $d_{c r}=d_{0}+0.1 d_{00}$. We also take $K / k=100$.

In Fig. 5 we see the final pattern of fractures for progressively deeper layers $n$, for a system of 1600 particles. As we see, the pattern of fractures that appears is highly disordered for the first few planes, with many fractures ending in the middle of the sample. When we go inside the material, there is a clear tendency to order, forming a polygonal pattern reminiscent of the experimental observations in basalts. Although in Fig. 5 some influence of the hexagonal structure chosen for the underlying lattice is observable, we have verified that the same qualitative process of ordering is found also for other underlying geometries, namely square. We have also looked at the final energy the pattern gets after fracturing, and this quantity is plotted in Fig. 6 as a function of $n$. As we see, this quantity has a tendency to be minimized as successive layers are fragmented, which is the right tendency predicted by our arguments. Moreover, in Fig. 6) we also plot the energy expected for a perfect hexagonal pattern, with the size of the hexagons chosen precisely in order to minimize the energy. We see that the solution that was found by the system was not the perfect one, but very close in energy to that. This is a further confirmation that the tendency to minimize the final energy is in fact the driving force for the formation of the polygonal pattern.

\section{PHENOMENOLOGICAL CALCULATION}

Having identified the reason why a superficially disordered pattern shows a tendency to order as it penetrates the material does not exhaust the interesting features of the problem. Here we will address the observation that patterns are usually seen to be polygonal, but not perfectly hexagonal, as it would be preferred by purely energetic reasons. We will show that this is a consequence of the minimization process, since the system is usually not able to reach the absolute minimum of the energy potential, but gets trapped in a relative minimum. Since the problem becomes computationally too costly to be tackled by the methods of the precedent section, we look for a phenomenological approach. We will need to calculate in some approximate manner the energy of the system as fractures advance, in order to search for fracture patterns that tend to minimize the energy.

A realistic calculation is rather complicated and it will be presented elsewhere. Here we will restrict to an heuristic analysis that however is able to show many of the known physical properties of fracture patterns.

Let us suppose that fractures divide the system in sectors of well defined areas $A_{i}$. We are interested in the elastic energy $E_{1}$ of the system after a vertical advance $d z$ of the fractures. To lowest order this energy must be a function of the $A_{i}$, of the elastic constants, and of the precise thermal state of the material. We will use the following expression

$$
E_{1}=E_{0}+\gamma \sum_{i} A_{i}^{\nu} d z,
$$

where $\gamma>0$ and $\nu>1$ are constants, and we have collected within $E_{0}$ all possible terms that do not depend on $A_{i}$. Three main facts have been used in constructing expression (2). First, the energy is an independent sum over different columns of terms that depend on $A_{i}$. This is the lowest order contribution we expect, in which we disregard contributions proportional to the particular form of the columns, and interaction terms between neighbor columns. Second, the final energy $E_{1}$ increases if $A_{i}$ increases (i.e., $\gamma>0$ ). This is the right tendency, since the final elastic energy becomes lower if new fractures are introduced in the system, and this implies a reduction of the typical $A_{i}$. Third, the exponent $\nu$ must be greater than one. This condition implies the tendency of the system to make the distribution of $A_{i}$ as uniform as possible in order to reduce $E_{1}$. With illustrative purposes, in the rest of this paper we will use $\nu=2$. We have repeated the simulations with $\nu$ in the 1.5 to 2.5 range with no significant change. The precise properties of the material an the thermal state of the system are contained in the value of $\gamma$.

Expression (2) for the final elastic energy has to be added with the change in the fracture energy $E_{2}$ during the vertical advance. This is simply given in terms of the energy needed to create the new fractures as

$$
\delta E_{2}=\eta L d z
$$

where $\eta$ is the fracture energy per unit area, and $L$ is the total length of fractures perpendicular to the propagation direction. Collecting the elastic (2) and fracture (3) energy terms, we can rephrase the minimum principle in the following form. Upon fracture advance, the energy functional

$$
\mathcal{E}=\gamma \sum_{i} A_{i}^{2}+\eta L
$$

tends to be minimized. The absolute minimum of (4) is attained by a perfect pattern of hexagons of side

$$
l_{\min }=(2 \eta / 9 \gamma)^{1 / 3}
$$

Now, we will use functional (4) to evolve irregular patterns (representing superficial fractures) up to point in 
which they stabilize, and then compare their statistical properties with real ones. Since we are not able to manage a completely general case, we chose a simple possibility that turns out to produce quite interesting results. We generate the pattern at the surface by a process of nucleation of linear fractures: from randomly chosen points within the plane we propagated two opposite, straight fractures. The process was repeated many times, with new fractures stopping as soon as they reached an older fracture. In Fig. [(a) we show a typical pattern generated by this process [18]. We simulate the modification of the pattern with an algorithm that makes small changes to the positions of the nodes at which fractures join. Each step in the modification of the pattern corresponds to the fracture pattern developing into the rock. The new position for a node was accepted if the new value of the energy, as given by Eq. (4) was lower than the previous value. In addition, at each step of the simulation the configuration was checked for the existence of very close nodes that can allow a change in the topology of the pattern according to the sketch of Fig. $7(\mathrm{~d})$. Again, the changes were accepted only if they reduce the value of $\mathcal{E}$. These processes are important since they change the number of sides of the polygons, and allow for a progress towards more stable patterns.

An intermediate pattern in the evolution process is shown in Fig. 7(b), and the final one (after which all proposed changes of the positions of the nodes increase the energy) is shown in Fig. 7(c). Since 'time' on our simulations corresponds to 'depth' in the rock, the ordering of our patterns represents the progressive order of the real lava fractures deeper into the rock [19]. The final pattern of Fig. 7(c) is not perfectly hexagonal, and thus it is only a relative minimum of (4). There is one single effective parameter in the simulation, that can be taken to be the side of the perfect hexagonal pattern of minimum energy $l_{\min }$. For our simulations this value, as given by (5), is indicated in Fig. 7. The qualitative similarity of the final pattern with that of the Giant's, Causeway shown in Fig. 1, is apparent. This polygonal pattern is now exposed at the surface of the rock, but there is evidence that this is not the original surface. In Fig. 8 we show two quantities that are a measure of the statistical similarity between our patterns and the real ones. In Fig. 8 (a) we see the results for the frequency of appearance of polygons with a given number of sides (in this case we also include the results on cornstarch by Müller [3] ), and in Fig. $8(b)$ the corresponding values for the mean area of polygons with a given number of sides, both in our simulations and in the real patterns. The configurations generated by our model are remarkably realistic. We see that, both in real cases and in our simulations, the fractures never reach a perfect hexagonal pattern. Instead, a reproducible distribution of polygons, most of them with 5,6 , and 7 sides is obtained, with a minor contribution of polygons with 4 and 8 sides. Also, polygons with higher number of sides have larger area as Fig. 8(b) shows.

\section{SUMMARY AND PERSPECTIVES}

In this paper we have given a first approach to a consistent model for the existence of columnar polygonal patterns in lava flows and some dessicating materials. We have shown in numerical simulations on a discrete model that fractures appear as irregular cracks at the free surface of the material and become ordered as they penetrate into the interior. We have argued that this effect is a consequence of a tendency to minimize an energy functional. The process of minimization follows a rough landscape, and is always towards a local minimun. This process is therefore monotonically decreasing with the thermal fluctuations playing no role. Relying on this principle, we showed that the statistical properties of experimental polygonal patterns can be reproduced.

There are still some problems that deserve further consideration and that we plan to discuss in a forthcoming publication. They have to do mainly with the realistic conditions of cooling. As discussed in section II, it is precisely the decreasing of the temperature gradient ahead of the fractures that makes possible the sequential advance of the fracture front, in a coordinated way all across the sample. The detailed study of this problem provides predictions for the width of chisel marks on the columns.

We also have to determine in a realistic situation the value of the constant $\gamma$ and $\nu$ in expression 2. This will allow to calculate in particular the typical values for the polygons in basalts and starches. Under realistic cooling conditions we also have to face the problem that temperature changes with time, and the effect of this on the advance of the fracture front has to be discussed.

We thank Roy Clarke, Eric Clement, Alan Cutler, Eric Essene, Len Sander and Youxue Zhang for very useful suggestions. A.G.R. acknowledges partial support from the National Science Foundation. E.A.J. acknowledges the hospitality of ICTP, Trieste, Italy, where part of this worked was done, and financial support from CONICET (Argentina).

[1] A. Holmes, Principles of Physical Geology (Wiley, New York, ed. 3, 1978), pp. 1-3.

[2] J. Walker, Sci. Am. 255, 178 (1986).

[3] G. Müller, J. Geophys. Res. 103, 15239 (1998).

[4] M. P. Ryan and C. G. Sammis, Geol. Soc. Am. Bull. 89, 1295 (1978).

[5] J. M. DeGraff and A. Aydin, Geol. Soc. Am. Bull. 99, 605 (1987)

[6] A. Aydin and J. M. DeGraff, Science 239, 471 (1988).

[7] R. Mallet, Philos. Mag. 50, 122 and 201 (1875); I. J. Smalley, Geol. Mag. 103, 110 (1966). 
[8] D. Weaire and S. Hutzler, The Physics of Foams (Clarendon, Oxford, 1999).

[9] We will use the language appropriate for the fracturing problem under thermal gradients. The arguments, however, apply also to the dessication problem in starches and other materials, by replacing 'temperature' by 'humidity concentration' and similarly with other expressions.

[10] A. Groisman and E. Kaplan, Europhys. Lett. 25, 415 (1994); P. Meakin, Science 252, 226 (1991); W. Korneta, S. K. Mendiratta, and J. Menteiro, Phys. Rev. E 57, 3142 (1998).

[11] K. A. Shorlin, J. R. de Bruyn, M. Grahan, and S. W. Morris, Phys. Rev. E 61, 6950 (2000).

[12] T. Hornig, I. M. Sokolov, and A. Blumen, Phys. Rev. E 54, 4293 (1996).

[13] K. Leung and J. V. Andersen, Europhys. Lett. 38, 589 (1997); S. Kitsunezaki, Phys. Rev. E, 60, 6449 (1999).

[14] L. B. Freund, Dynamic Fracture Mechanics (Cambridge University Press, New York, 1990).

[15] L.D. Landau and E.M. Lifshitz, Theory of Elasticity (Butterworth-Heinemann, Oxford, ed. 3, 1986), Ch. 1.

[16] In two dimensional dessication experiment, an evenly spaced set of fractures is usually obtained (see [17 and [11). Our minimum principle gives a natural justification to this fact.

[17] C. Allain, and L. Limat, Phys. Rev. Lett. 74, 2981 (1995).

[18] This procedure exaggerates the disorder in the first layer. We have repeated our simulations starting with a pattern consisting of 90 degrees junctions only, with identical results.

[19] In order that the final pattern be independent of the initial density of fractures, this has to be high enough. This is because we do not allow for the creation on new fractures in the algorithm, all of them have to be there 'from the beginning'.

[20] P. Budkewitsch, P. Robin, J. Volcanol. Geotherm. Res. 59, 219 (1994).

[21] J. P. O'Reilly, Trans. R. Irish Acad. 26, 641 (1879).

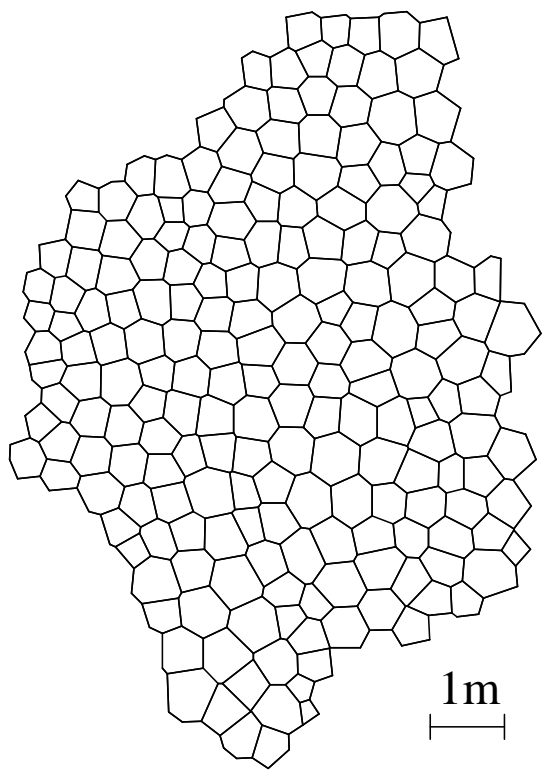

FIG. 1. Polygonal pattern seen perpendicularly to some of the columns of the Giant's Causeway, a Tertiary lava flow in Antrim, Northern Ireland, from Ref. 20] (originally from a map by O'Reilly 21])).

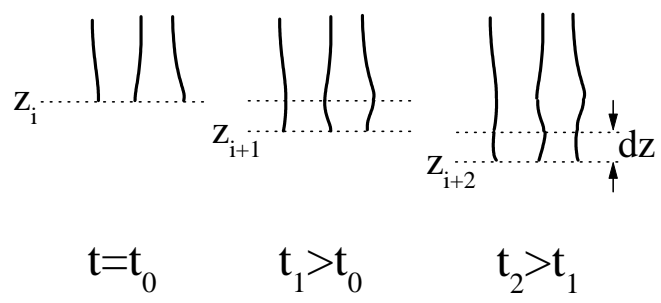

FIG. 2. Schematic representation of sequential fragmentation in a two-dimensional geometry . Continuous lines represent the advancing fractures. At each time step a layer of material of thickness $d z$ fractures. New fractures appear below those already present, but slight modifications in their positions are possible, and in fact crucial to the ordering process. 


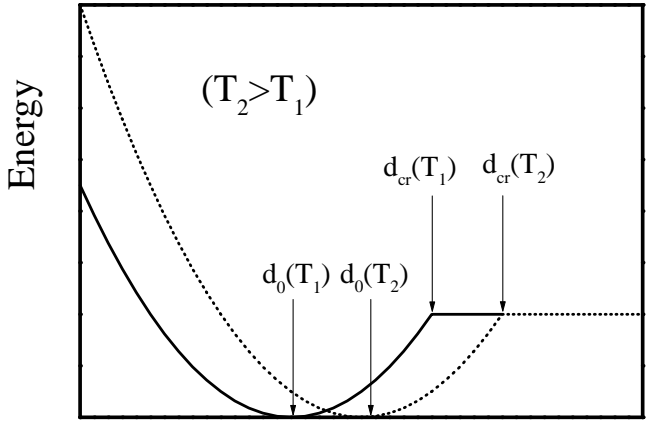

distance

FIG. 3. Schematic energy vs. distance curve for neighbor particles in the discrete model of the rock. There is an equilibrium distance between particles that depends on temperature $d_{0}(T)$. Deviations from this distance costs an elastic energy which is quadratic in the displacement. If the distance becomes greater than some critical distance $d_{c r}(T)$ then energy saturates. In this way we model fractures, since in this range there is no force between particles.

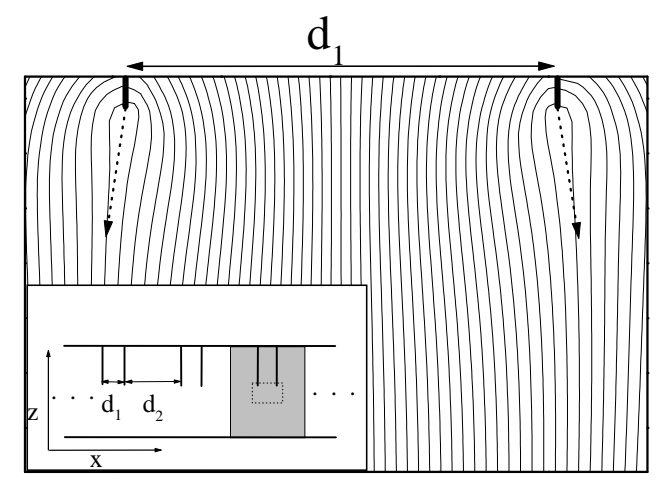

FIG. 4. Stresses ahead of an unevenly set of fractures as depicted in the inset. In the main figure we plot the stress field in the neighborhood of a couple of close fractures (see text for details). Directions of maximum stress at the tips of the fractures are indicated by the arrows. The simulated box is marked in gray in the inset. Periodic boundary conditions are used along $x$, and free boundary conditions along $z$. The dotted box in the inset is the region plotted in the main figure. We have used $d_{2} / d_{1}=4$.
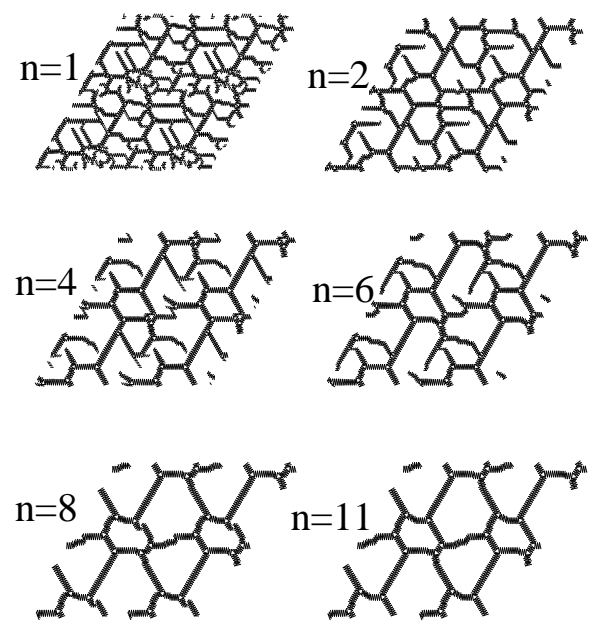

FIG. 5. The final patterns of fractures for progressively deeper layers $n$ in a sequential fragmentation process for layers with 1600 particles. For clarity reasons, in the plots the system has been duplicated both in horizontal and vertical direction (periodic boundary conditions are used). In the plots, each thick fracture is formed by small lines mostly perpendicular to the fracture that join the ends of springs that have failed after a contraction up to 0.89 of the original distance between lattice points.

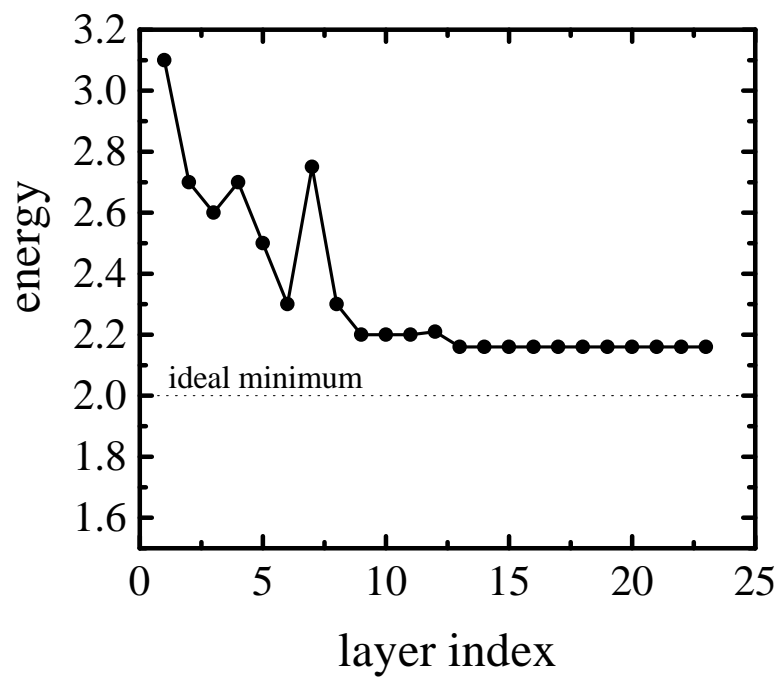

FIG. 6. Energy per particle of the pattern obtained in the simulations shown in the preceding figure, as a function of the layer index. The ideal minimum of a size-optimized honeycomb lattice is also shown. Energy is given in units of $10^{-3} K d_{00}^{2}$. 


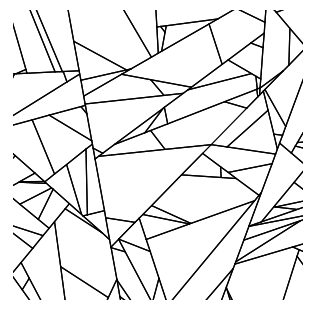

(a)

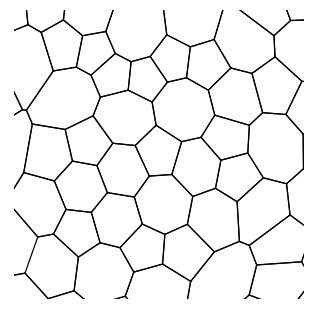

(c) $1_{\text {min }}$

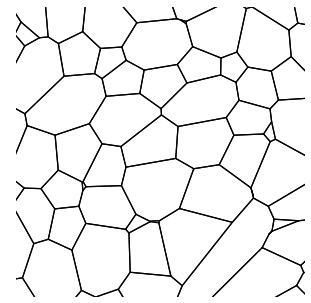

(b)

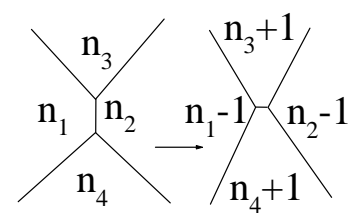

(d)

FIG. 7. Numerically evolved patterns of fractures. We see the original pattern (a), the final (stable) one (c), and one intermediate configuration (b). To avoid spurious edge effects, only the central region of a simulation performed on a larger sample is shown. The numerical algorithm is described in the text. Below the final pattern (c), the side of hexagons $l_{\text {min }}$ in the expected ideal honeycomb lattice is indicated. In (d) we see the kind of processes that allow for a change in the number of sides of adjacent polygons.

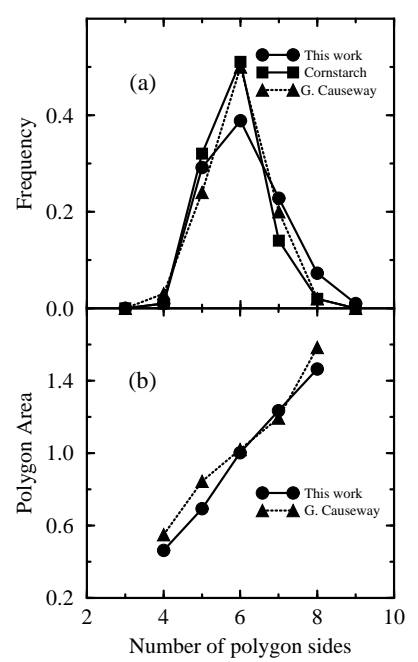

FIG. 8. (a) Histogram for the relative frequency (normalized to one) of appearance of polygons with different number of sides for the Giant's Causeway, columns in cornstarch [3], and from our simulations (an average over 10 final configurations as that of Fig. F(c) is shown). (b) Areas of the polygons normalized to the average area for polygons with different number of sides (data on cornstarch are not available). 